\title{
Model migration for CFD and verification of a six-rotor UAV downwash
}

\author{
Shenghui Yang ${ }^{1}$, Qing Tang ${ }^{2}$, Yongjun Zheng ${ }^{1 *}$, Xingxing Liu ${ }^{1}$, Jian Chen ${ }^{1}$, Xiaolong $\mathrm{Li}^{1}$ \\ (1. College of Engineering, China Agricultural University, Beijing 100083, China; \\ 2. National Research Centre of Intelligent Equipment for Agriculture, Beijing 100097, China)
}

\begin{abstract}
Currently, Computational Fluid Dynamics (CFD) has been used to investigate agricultural UAV downwash. However, the validations of CFD models are difficult to deal with. Current verification methods are to use either water-sensitive papers or wind-speed arrays, which could get wind distribution or speed only. In this study, model migration was used to develop and verify downwash CFD models. The basic idea is to try to use the results of a scaled-down drone to represent that of a real-used UAV. The CFD models of both a real-used six-rotor UAV, JF01-10, and a 1:10 scaled-down small drone were developed by ANSYS. Then, the scaled-down drone was utilized to conduct trials by particle image velocimetry (PIV), so that not only distribution and speed but also flowing direction of downwash could be obtained. Results indicated the relative error between the PIV tests and the CFD models of the small UAV was less than $12 \%$, while that between the tests and the CFD models of JF01-10 was less than 34\%. It could be indicated that model migration could reflect multiple downwash characteristics but should be optimized in some complex details. This study was a preliminary but fundamental attempt to investigate CFD modelling and validation of agricultural UAVs and provided a novel thinking of downwash verification.
\end{abstract}

Keywords: multi-rotor UAV, downwash, numerical simulation, computational fluid dynamics, verification, model migration DOI: $10.25165 /$ j.ijabe.20201304.5569

Citation: Yang S H, Tang Q, Zheng Y J, Liu X X, Chen J, Li X L. Model migration for CFD and verification of a six-rotor UAV downwash. Int J Agric \& Biol Eng, 2020; 13(4): 10-18.

\section{Introduction}

Multi-rotor Unmanned Aerial Vehicles (UAVs) specialized for plant protection have commonly used in agriculture throughout the world, especially in China ${ }^{[1,2]}$, and pesticide spray is one of their key functions. A large amount of research focused on the distribution of droplets during UAV operation ${ }^{[3-10]}$, like testing UAV performance and finding out optimal parameters for operation. For instance, Liao et al examined three kinds of UAVs, including a four-rotor, a six-rotor and an eight-rotor UAV, to obtain their spray swath and uniformity. In addition, different factors, such as flight velocities and heights, were employed in the tests so as to analyze the impact of the variables on spraying ${ }^{[4]}$. Wang et al. ${ }^{[5]}$ also varied different factors of UAVs to spray pineapple. Optimal parameters for spraying were found out. However, it should be pointed out that downwash airflow formed by UAV propellers is the essential issue to strongly affect droplet distribution since it can directly carry the sprayed pesticide droplets to penetrate canopies and attach leaves ${ }^{[2]}$ so as to ensure spray effectiveness. Furthermore, downwash airflow has two obvious characteristics, one, wind speed, the other, wind direction. Both

\section{Received date: 2019-12-04 Accepted date: 2020-03-25}

Biographies: Shenghui Yang, PhD, research interests: mechatronics, applied agricultural aviation, Email: yshgxy@cau.edu.cn; Qing Tang, PhD, Assistant Professor, research interests: applied agricultural aviation, plant protection, Email: tqmonk@163.com; Xingxing Liu, PhD, Associated Professor, research interests: mechatronics, electric and control, Email: liuxingxing56285@ 163.com; Jian Chen, $\mathrm{PhD}$, Associated Professor, research interests: agricultural aviation, navigation and control, Email: jchen@cau.edu.cn; Xiaolong Li, Master, research interests: mechatronics, Email: 17545569054@163.com

*Corresponding author: Yongjun Zheng, PhD, Professor, research interests: intelligent precision agricultural equipment. College of Engineering, China Agricultural University, Beijing 100083, China. Tel: +86-10-62736385, Email: zyj@cau.edu.cn. of them could influence the droplet movement. Therefore, it is necessary to analyze the specifications of the downwash airflow of UAVs.

Wang et al. ${ }^{[8]}$ proposed a method of spatial pesticide spraying deposition quality balance to analyze downwash impact. They mainly used image processing based on water-sensitive paper to calculate and invert distribution results. However, the results using water-sensitive paper may be affected by several factors, such as air humidity and image areas ${ }^{[1]}$, and cannot display the downwash distribution in detail. Computational fluid dynamics (CFD) could deal with these limitations. It is able to clearly show the flowing and distributing of downwash in detail with the values of wind speed and wind direction. At present, a few studies have exploited CFD to simulate the downwash airflow of multi-rotor UAVs as well as fixed-wing $\mathrm{UAVs}^{[12]}$ in agriculture. Nevertheless, it is difficult for real multi-rotor UAVs to deal with the verification of downwash CFD models, because of the large size of UAVs, the wide distribution of downwash and the different directions of downwash flowing. Especially, the research on CFD related to agricultural aviation is just in its staring. For instance, Yang et al presented the numerical simulation of a real six-rotor $\mathrm{UAV}^{[13]}$, but there was no verification of the CFD models, except the trials only for nozzle simulation. Ling et al presented the simulation of a type of UAV using the CFD method ${ }^{[14]}$, but there was no verification, either.

Some scholars attempted to validate the CFD models in their research. The commonly used approach was to apply windspeed sensors ${ }^{[15-17]}$, sometimes still to lay water-sensitive paper ${ }^{[18]}$. Although the wind speed data and fitted curves could show the wind variation of downwash to obtain a few distribution laws, the flowing direction and specifications of downwash could not be verified.

Particle image velocimetry (PIV) is an accurate way to verify fluid flow due to a large number of spatial points recorded in the 
same transient. Only a few studies in agricultural aviation have utilized this method ${ }^{[19]}$. On one hand, flowing speed and direction could be indeed acquired and displayed in detail. On the other hand, PIV could not be used in an outdoor environment or for the verification of the downwash of real-entire UAVs because of its limitation of collected image areas and light intensity. Thus, if PIV could be somehow exploited for indoor UAV examinations, downwash details could be supplemented.

There are several methods to simplify real tests for the indoor environment. Among these, model migration has been widely used in the design and verification of aircraft ${ }^{[20,21]}$, meaning that the scaled-down model with highly similar shape and configuration can be exploited to replace the real aircraft for experiments ${ }^{[20]}$. The costs of both time and funds could be decreased. Therefore, model migration could deal with the difficulty of verification of the downwash CFD models of real UAVs. If model migration combined with PIV could be analogized to verify the downwash airflow of agricultural UAVs, it would be an appropriate and convenient means to obtain accurate results in the indoor environment to invert the downwash distribution of real-used UAVs.

Therefore, this paper presents the verification of downwash CFD models of a six-rotor UAV (JF01-10), a commonly used type of drone, based on the analogy of model migration. ANSYS was utilized to simulate the downwash of both the real six-rotor UAV and a scaled-down drone. Moreover, PIV was used to obtain the accurate downwash distribution of the scaled-down drone in detail. Then, the test results would be compared with the downwash CFD of both the scaled-down drone and the real UAV to make conclusions. This investigation is a significant attempt to deal with the difficulty of CFD verification and could be a reference for further related studies in agricultural aviation.

\section{Materials and methods}

\subsection{Model migration}

In terms of model migration, a highly similar model could represent the real aircraft. Thus, in mathematics, it is a process that uses unified linear transform (as shown in Equation (1)) from a basic model (the real UAV) to a new one (the scaled-down model). Any point on the surface of one model can be found on that of the other $^{[20]}$

$$
\left[\begin{array}{l}
x_{n} \\
y_{n} \\
z_{n}
\end{array}\right]=A\left[\begin{array}{l}
x_{b} \\
y_{b} \\
z_{b}
\end{array}\right]+B=\left[\begin{array}{lll}
a_{11} & a_{12} & a_{13} \\
a_{21} & a_{22} & a_{23} \\
a_{31} & a_{32} & a_{33}
\end{array}\right]\left[\begin{array}{l}
x_{b} \\
y_{b} \\
z_{b}
\end{array}\right]+\left[\begin{array}{l}
b_{1} \\
b_{2} \\
b_{3}
\end{array}\right]
$$

where, $\left[x_{b}, y_{b}, z_{b}\right]^{\mathrm{T}}$ is the point on the basic model; $\left[x_{n}, y_{n}, z_{n}\right]^{\mathrm{T}}$ is the point on the new model; $A$ and $B$ are solvable linear constant matrices.

Due to the symmetrical structure of six-rotor UAVs, symmetric planes can be firstly linearly fitted, as shown in Equation (2).

$$
\left[\begin{array}{l}
x_{n} \\
z_{n}
\end{array}\right]=A_{1}\left[\begin{array}{l}
x_{b} \\
z_{b}
\end{array}\right]+B_{1}=\left[\begin{array}{ll}
a_{11} & a_{13} \\
a_{31} & a_{33}
\end{array}\right]\left[\begin{array}{l}
x_{b} \\
z_{b}
\end{array}\right]+\left[\begin{array}{l}
b_{1} \\
b_{3}
\end{array}\right]
$$

Then, the points of different directions truncated by a line parallel to different axis can be used to calculate and evaluate the similarity of the two models.

Based on the points mentioned above, the core of model migration is to scale the basic model in proportion. Therefore, model migration was analogized in this study so that the scaled-down linear transform was applied.

\subsection{Materials and devices}

\subsubsection{Particle image velocimetry}

A high-speed particle image velocimetry (PIV) was used for the indoor test. It is a kind of non-interactive measurement method to obtain the velocity field of a certain area. Firstly, tracer particles with proper sizes were injected into the test section. They were not only big enough to reflect intense laser but also small enough to follow the movement of the air. The definition of their size is mentioned later. Two laser sheets have been illustrated a region of interest in a short-time gap and a high-speed camera was exposed twice to capture the two images of the tracer particles in this area. Due to the time difference of capturing these two images, the movement of the tracer particles could be used to calculate the velocity field at the moment. Figure 1 shows more details about the PIV principle.

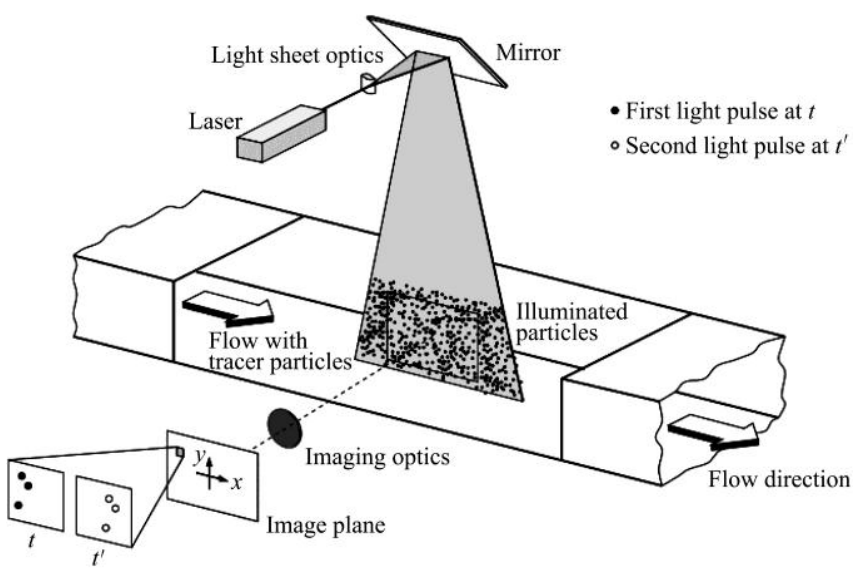

Figure 1 Schematic diagram of the PIV method ${ }^{[22]}$

After obtaining a pair of particle images, the calculation of velocity vectors was made by Fourier transformation. The cross-correlation value is

$$
R_{\mathrm{II}}(x, y)=\sum_{i=-K}^{K} \sum_{j=-L}^{L} I(i, j) I^{\prime}(i+x, j+y)
$$

where, $K$ and $L$ are the edge length of the interrogation window and the variables; $I$ and $I^{\prime}$ are the samples (e.g. intensity values) as extracted from the images, where $I^{\prime}$ is larger than the template $I$ and essentially the template $I$ is linearly 'shifted' around in sample $I$ ' without extending over edges of $I^{\prime}$.

For each choice of a sample shift $(x, y)$, the sum of the products of all overlapping pixel intensities produces one cross-correlation value $R_{\mathrm{II}}(x, y)$. Then, the highest value in the correlation plane can be used to detect a correlation peak and obtain a subpixel accurate displacement estimate of its location:

Step 1: Scanning the correlation plane $R=R_{\mathrm{II}}$ for the maximum correlation value $R(i, j)$ and storing its integer coordinates $(i, j)$.

Step 2: Extracting the adjoining four correlation values, $R(i-1, j), R(i+1, j), R(i, j-1)$ and $R(i, j+1)$.

Step 3: Using three points in each direction to apply the three-point estimator that is generally a Gaussian curve. The formulas for Gaussian function is

$$
\left\{\begin{array}{l}
x_{0}=i+\frac{\ln R_{(i-1, j)}-\ln R_{(i+1, j)}}{2 \ln R_{(i-1, j)}-4 \ln R_{(i, j)}+2 \ln R_{(i+1, j)}} \\
y_{0}=j+\frac{\ln R_{(i, j-1)}-\ln R_{(i, j+1)}}{2 \ln R_{(i, j-1)}-4 \ln R_{(i, j)}+2 \ln R_{(i, j+1)}}
\end{array}\right.
$$

The PIV system used was powered by LAVISION, as shown in Figure 2. This kind of system incorporated a high-speed 
CMOS camera (1280-1,024 pixels at $2000 \mathrm{fps}$ ) with matched double-pulsed Nd:YLF laser (20 mJ/pulse, $527 \mathrm{~nm}$ wavelength). Convex lenses were arranged to form a quasi-parallel laser sheet with a beam waist thickness of $1 \mathrm{~mm}$. A $105 \mathrm{~mm}$ lens (set to an f-stop of 2.8) was used to obtain a field of view about $300 \mathrm{~mm} \times 300$ $\mathrm{mm}$. Moreover, a Laskin nozzle seeder was used with Di-2-Ethylhexyl-Sebacat (DEHS) as the substrate and it generated a fine mist with a nominal particle diameter of $1 \mu \mathrm{m}$. The relative density and size of particles are known to be good for the PIV test in the air ${ }^{[22]}$.

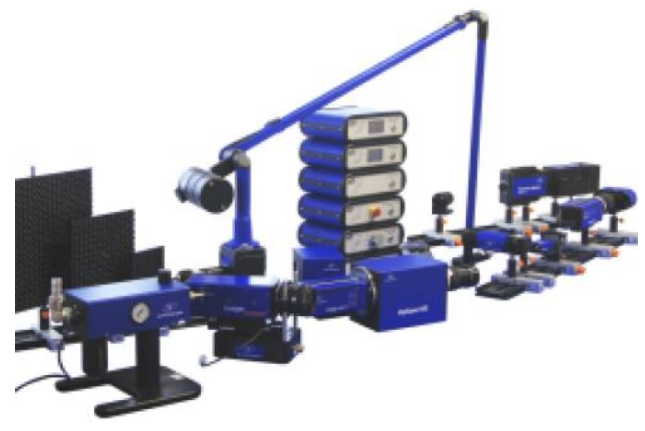

Source: From http://www.lavision.de

Figure 2 PIV used in the tests

\subsubsection{Unmanned aerial vehicles}

A real-used six-rotor UAV, JF01-10, was the basic aircraft in this study, as shown in Figure 3. Its width is about $1780 \mathrm{~mm}$ and the propeller is 22 inches (about $558 \mathrm{~mm}$ ).

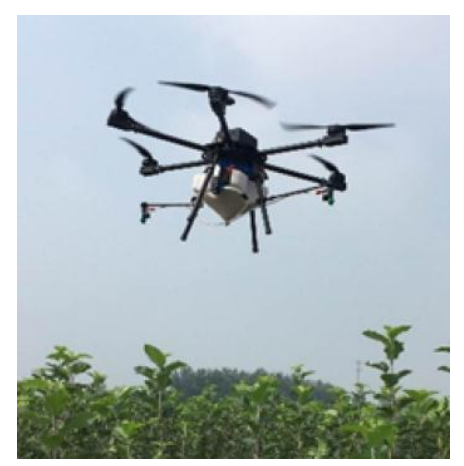

Figure 3 Real-used six-rotor UAV, JF01-10

Due to the limit of both indoor test areas and the image size acquired by the PIV, JF01-10 could not be directly used for data acquisition by PIV. Thus, compared with the size of JF01-10, a 1:10 scale-down drone was used (as shown in Figure 4). The propeller shields of the small six-rotor drone were cut off in order to keep key similarities to JF 01-10 before the trials. The size of this drone excluding the shields was about $176 \mathrm{~mm}$, very closed to the ten-time smaller size of JF01-10. The propeller was about $55 \mathrm{~mm}$ length, also really closed to the scale-down requirement of JF01-10 propeller. The heights of both CFD simulation and verification were also reduced by 10 times.

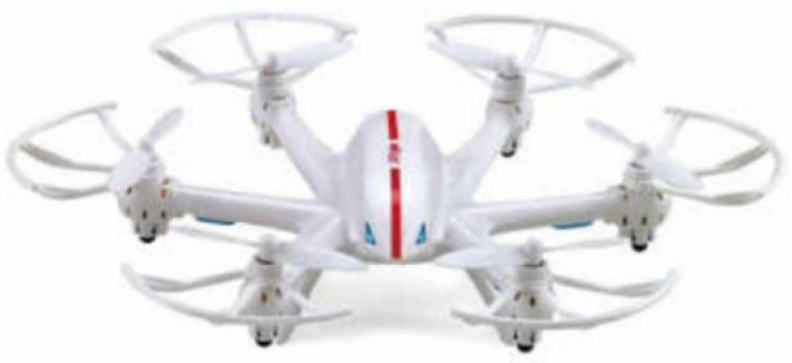

Figure 4 Small Scaled-down six-rotor UAV

\subsection{CFD models by using ANSYS}

Three-Dimensional (3D) physical models of both the real-used UAV, JF01-10 (Figure 5a), and the scaled-down drone (Figure 5b) were established by SolidWorks. Each part was simplified but kept key features to reduce computation meshes. The entire simulation domain consisted of rotational domains and a non-rotational domain. The rotational domains based on propellers were established as a cylinder (Figure 5c) while the part outside the rotational domains was the non-rotational domain.

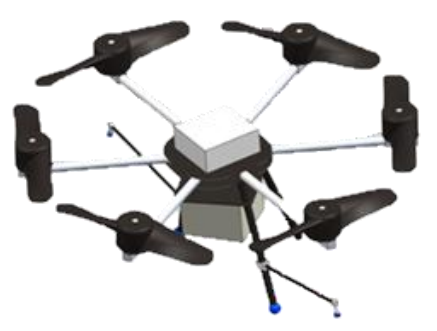

a. JF01-10 3D model

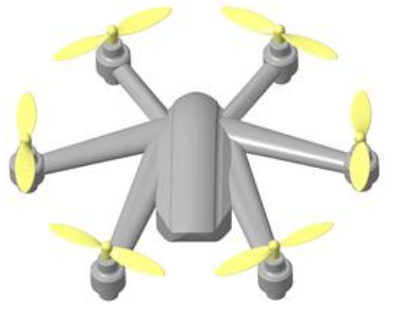

b. Small drone 3D mode

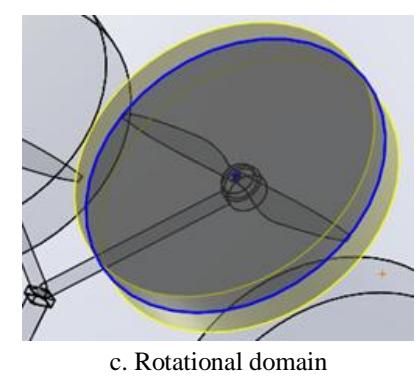

Figure 53 D real-used physical models

ANSYS was used to develop the CFD models of both the real-used UAV and the scaled-down drone. In terms of the real-used UAV CFD models, Figure 6 shows the CFD results of the real-used UAV by the previous study ${ }^{[23]}$. Mesh in Workbench was used for meshing and Fluent in Workbench was used as the solver. The unstructured tetrahedral mesh was applied and the size of the grids was the default. The grids on the curved surface were densified. SST $k-\varepsilon$ model was employed. A single central processing unit (CPU) of a work station, Think Station P910, was used.

According to Figure 6, the airflow velocities of the downwash were $12.43 \mathrm{~m} / \mathrm{s}, 12.59 \mathrm{~m} / \mathrm{s}$, and $12.59 \mathrm{~m} / \mathrm{s}$ respectively. In terms of the scaled-down drone CFD models, Mesh in Workbench was exploited for meshing and Fluent in Workbench was applied as the solver. The entire fluid domain contains six rotational domains and a non-rotational domain, ten times than the propeller-included wheelbase of the scaled-down drone. The unstructured mesh was utilized with the size function 'Curvature'. The size of the grids was the default since the minimum size of grids of this default setting was $1.4689 \times 10^{-3} \mathrm{~m}$ while the minimum size of the small drone was $1.5 \times 10^{-3} \mathrm{~m}$. Thus, the default setting could meet the requirement. The grids on the curved surface were densified in double. The number of nodes was 102207 and that of elements was 518834 .

Moreover, Steady, Pressure-based and Absolute Velocity Formulation were set for the steady CFD simulation. The $k-\varepsilon$ model was employed. Fluid material was set as air. Then, in Cell Zone Conditions, the rotating speed was set as $1500 \mathrm{r} / \mathrm{min}$ and each neighbor propeller turned in the opposite direction. For Boundary conditions, the interface between the rotational domains and the non-rotational domain was set as a fixed-rotational interface. A couple of solvers was used. 1000 interactions were set. Other boundary conditions and initial conditions were default. 
Figure 7 shows the CFD model of the downwash of the scaled-down drone. According to Figure 7, the airflow velocities of the downwash of the small UAV were $1.9 \mathrm{~m} / \mathrm{s}$ and $1.8 \mathrm{~m} / \mathrm{s}$, respectively.

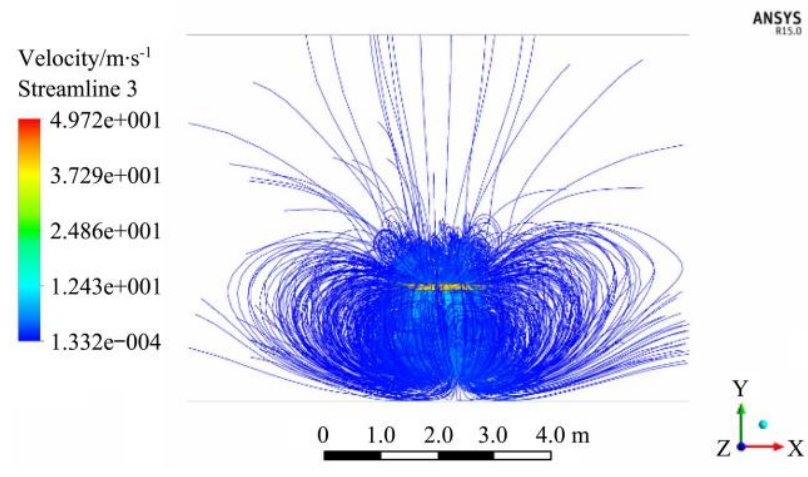

a. Velocity distribution of $2000 \mathrm{~mm}$ simulation height

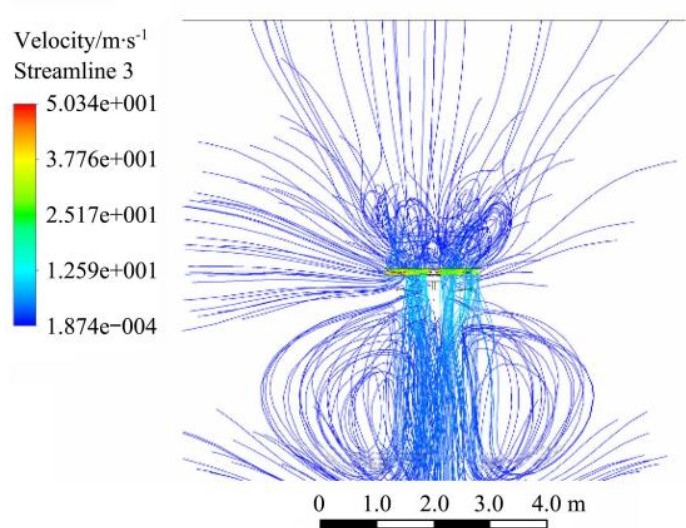

c. Velocity distribution of $5000 \mathrm{~mm}$ simulation height

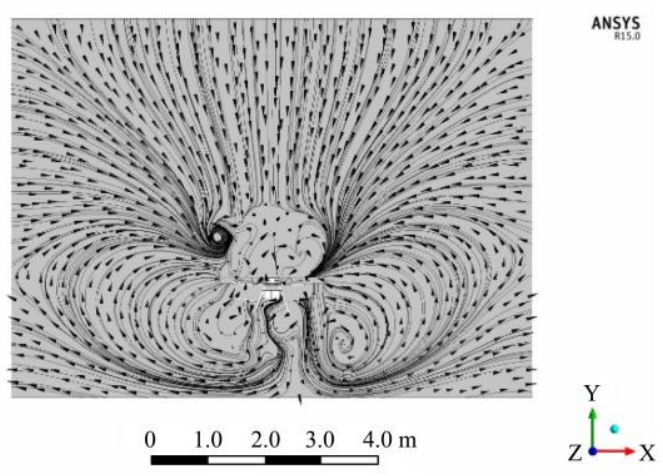

b. Flowing direction of $2000 \mathrm{~mm}$ simulation height

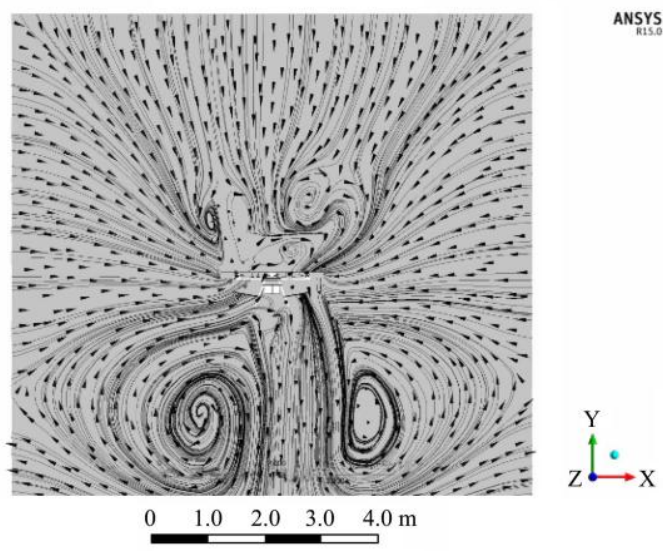

Figure 6 Downwash CFD of JF01-10 by ANSYS

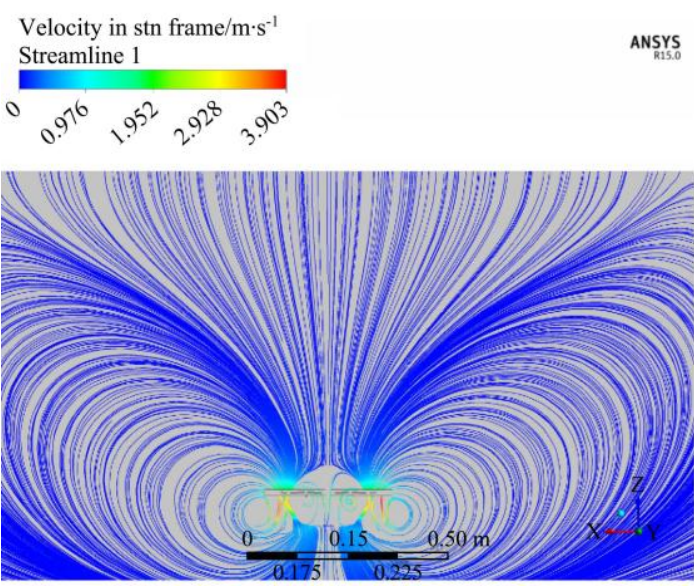

a. Velocity distribution of $200 \mathrm{~mm}$ simulation height

Velocity in stn frame $/ \mathrm{m} \cdot \mathrm{s}^{-1}$ Streamline 1

$0.90^{85} \quad 9^{90} 2^{99^{5}} \quad 39^{x^{0}}$

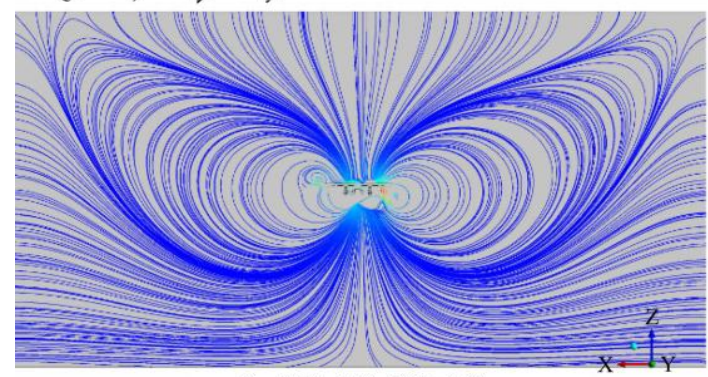

$\begin{array}{lllll}0 & 0.15 & 0.30 & 0.45 & 0.60 \mathrm{~m}\end{array}$

c. Velocity distribution of $500 \mathrm{~mm}$ simulation height d. Flowing direction of $5000 \mathrm{~mm}$ simulation height

ANSYS

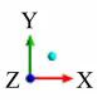

S15.0

Pressure $/ \mathrm{Pa}$

Contour 1

ANSYS

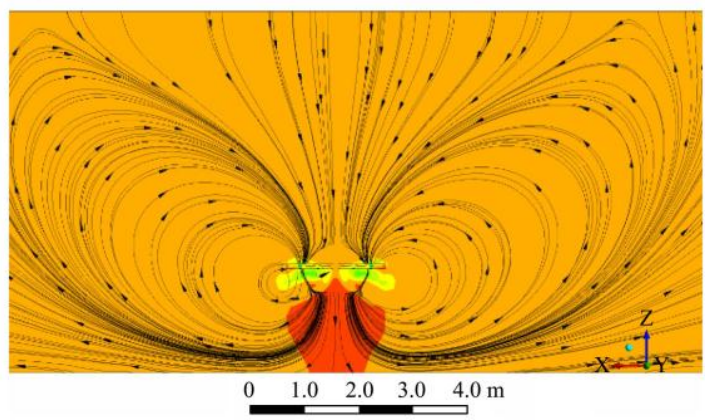

b. Flowing direction of $200 \mathrm{~mm}$ simulation height

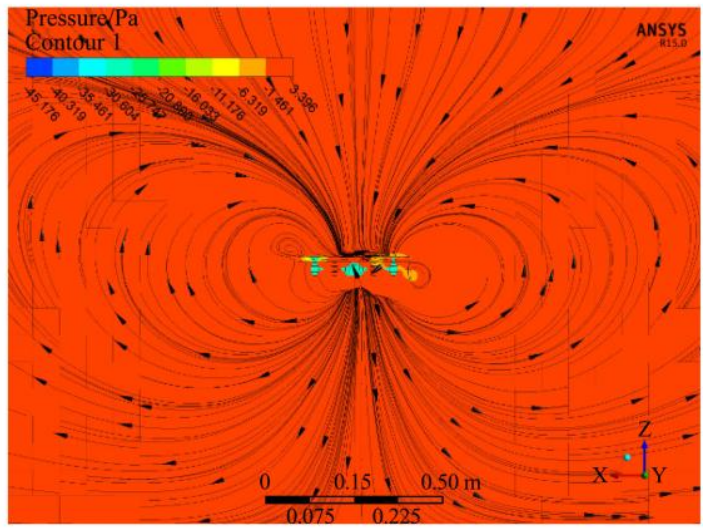

d. Flowing direction of $500 \mathrm{~mm}$ simulation height

Figure 7 Downwash CFD of the scaled-down drone by ANSYS 


\section{Verification of CFD models based on model migration}

\subsection{Experimental site}

The experiments were conducted at the Agricultural Aerial Spray Aerodynamic Lab in Xiaotangshan National Precision Agriculture Base (Beijing, China), from March 25-28, 2019.

Figure 8 shows the experimental site for verification. The scaled-down drone was hung and fixed on the bracket. PIV was used throughout the trials. Moreover, a large piece of stainless board with a mirror surface was used to concentrate the laser sheet to increase the intensity of the region of interest.

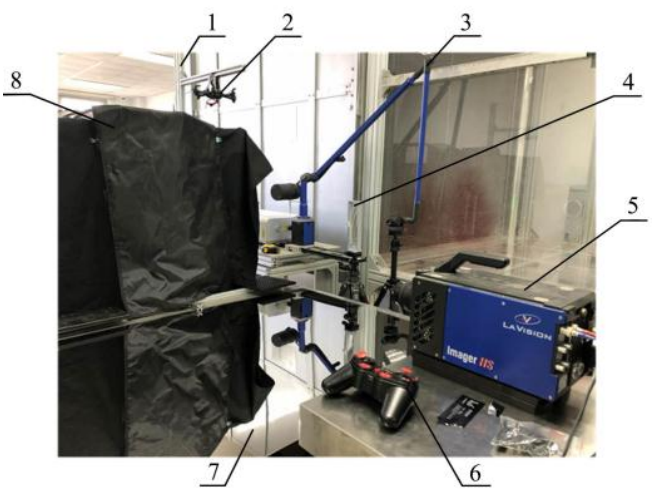

1. Bracket 2. Small six-rotor UAV 3. PIV laser source 4. Spectroscopic lens 5. PIV image collector 6. Remote control 7. Mirror panel 8. Black background

Figure 8 Experimental site for verification

As shown in Figure 9, the distance between the drone and the

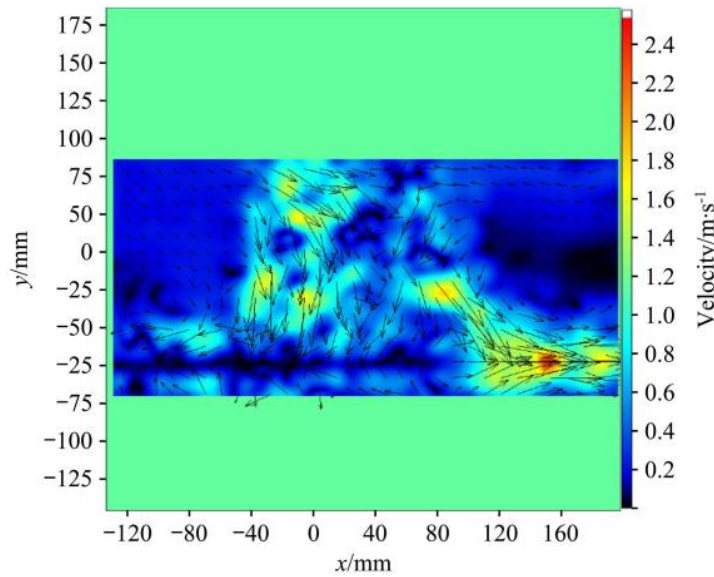

a. Frame examples of the $200 \mathrm{~mm}$-height tests of the scaled-down drone
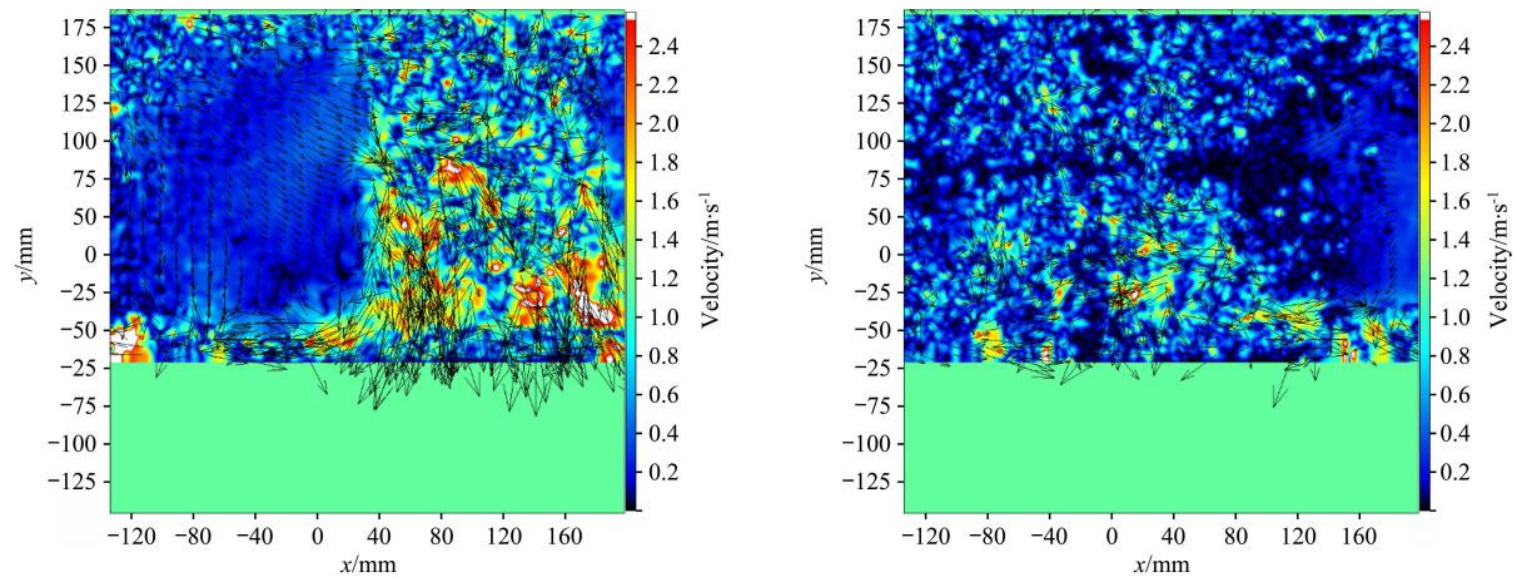

b. Frame examples of the $500 \mathrm{~mm}$-height tests of the scaled-down drone

Figure 10 Frame examples of the scaled-down UAV from about 1000 frames by the image collector mirror surface was $200 \mathrm{~mm}$ and $500 \mathrm{~mm}$ respectively. Due to the limit of the image area by PIV, the trial of $500 \mathrm{~mm}$ height was separately shot from left to right and top to bottom. During the tests, the time interval between two frames was $1 \mathrm{~ms}$ and the maximum moving distance of particles was less than $5 \mathrm{~mm}$. Thus, the interrogation window was set as $32 \times 32$ pixels (a square of $10 \mathrm{~mm} \times 10 \mathrm{~mm}$ ) to keep particle moving distance less than $1 / 4$ of the interrogation window length ${ }^{[22]}$. The overlap ratio was set as $50 \%$, so the relative spatial resolution of the velocity field was $5 \mathrm{~mm}$. a. Test of $200 \mathrm{~mm}$

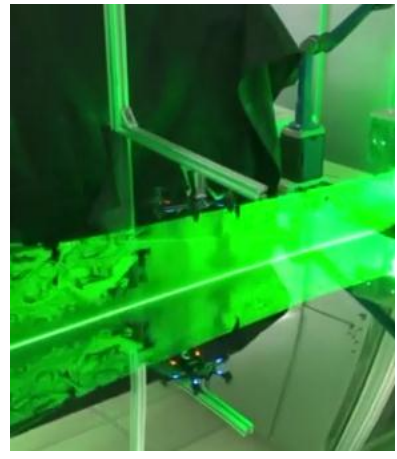

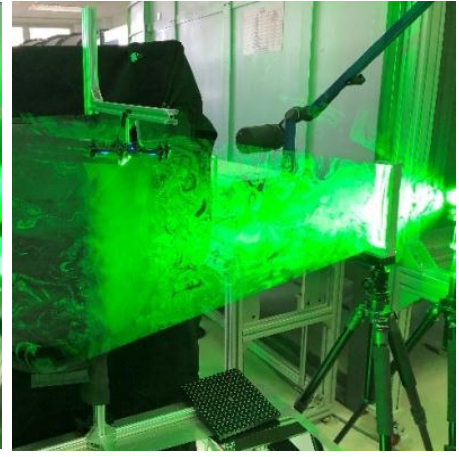

b. Test of $500 \mathrm{~mm}$
Figure 9 Test process by using the PIV

\subsection{Experimental results}

Figure 10 shows some frames by the image collector. All the frames, about 1000 pictures in total over each test, were used to be processed for results.

Figure 11 and Figure 12 respectively show the results of the $200 \mathrm{~mm}$ test and the $500 \mathrm{~mm}$ tests, the redder the color, the faster the velocity.

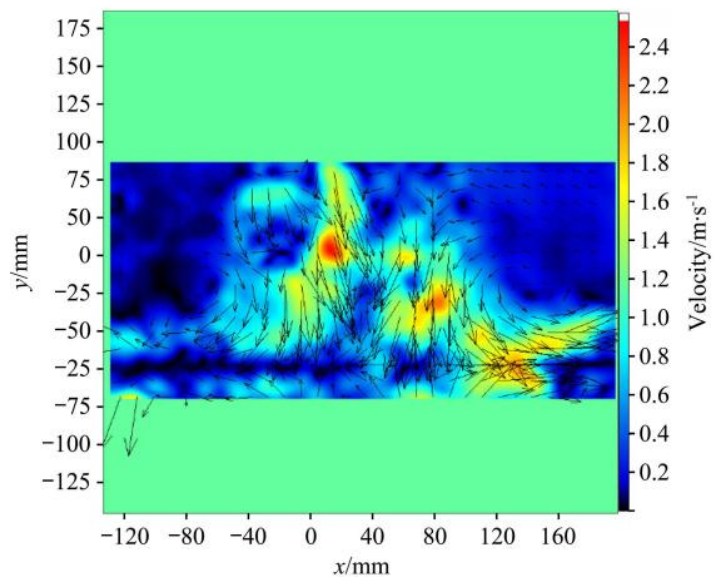




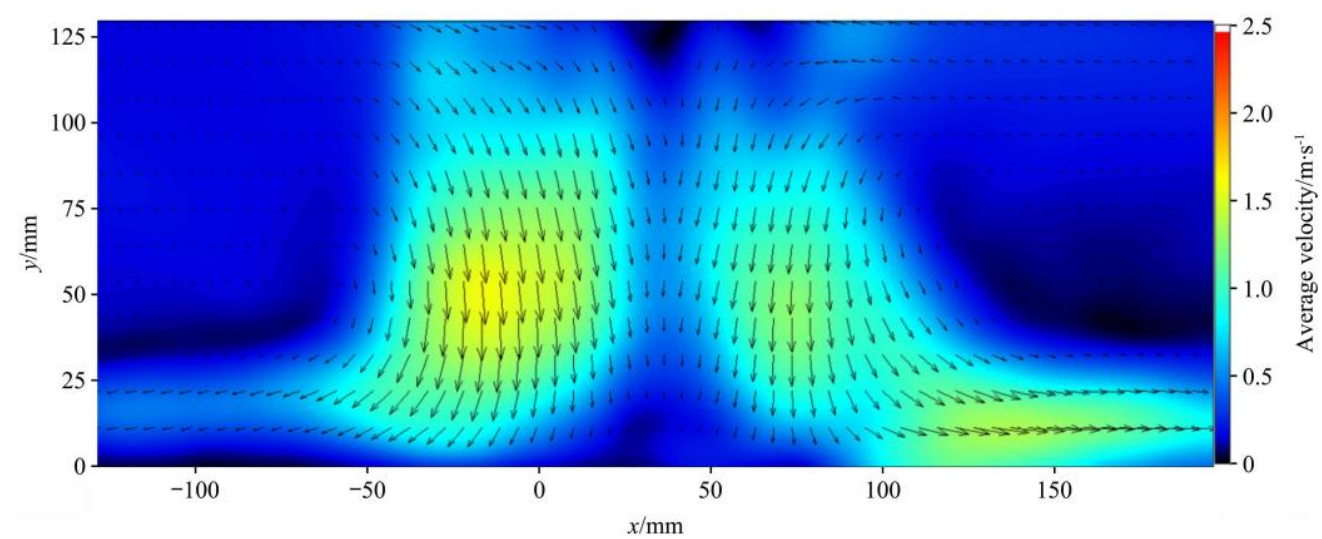

a. Velocity and distribution of downwash in the $200 \mathrm{~mm}$ tests
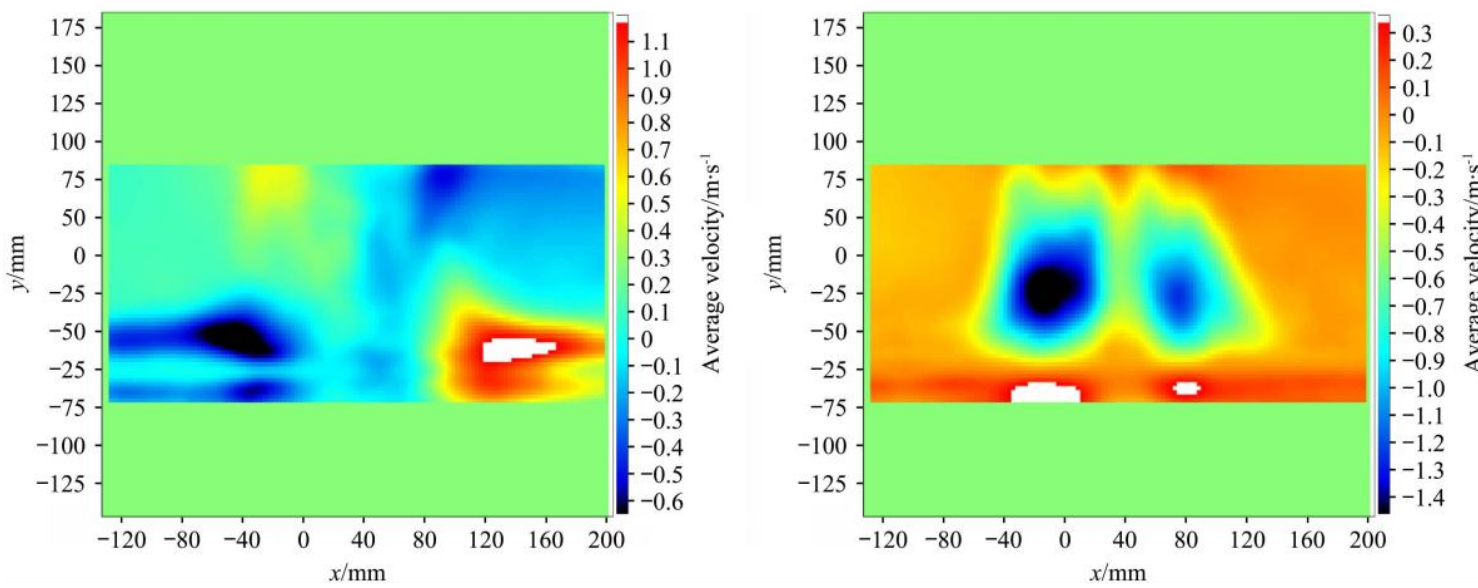

b. Average velocity of $V_{x}$ in the $200 \mathrm{~mm}$ tests

Figure 11 Results of the $200 \mathrm{~mm}$ PIV tests of the scaled-down UAV

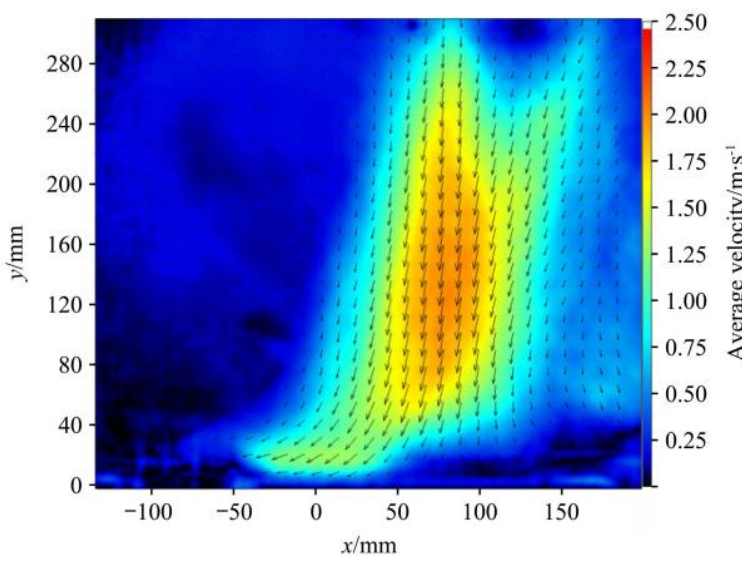

a. Left of the $500 \mathrm{~mm}$ PIV tests of the scaled-down drone

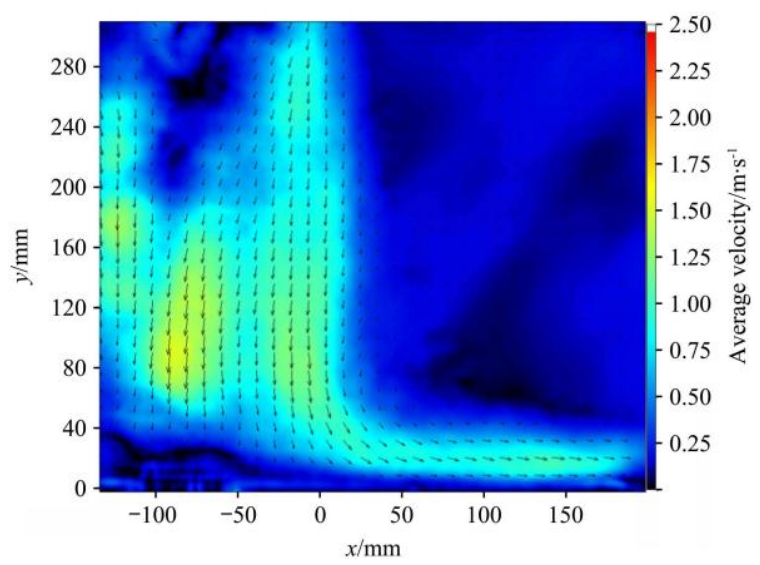

b. Right of the $500 \mathrm{~mm}$ PIV tests of the scaled-down drone

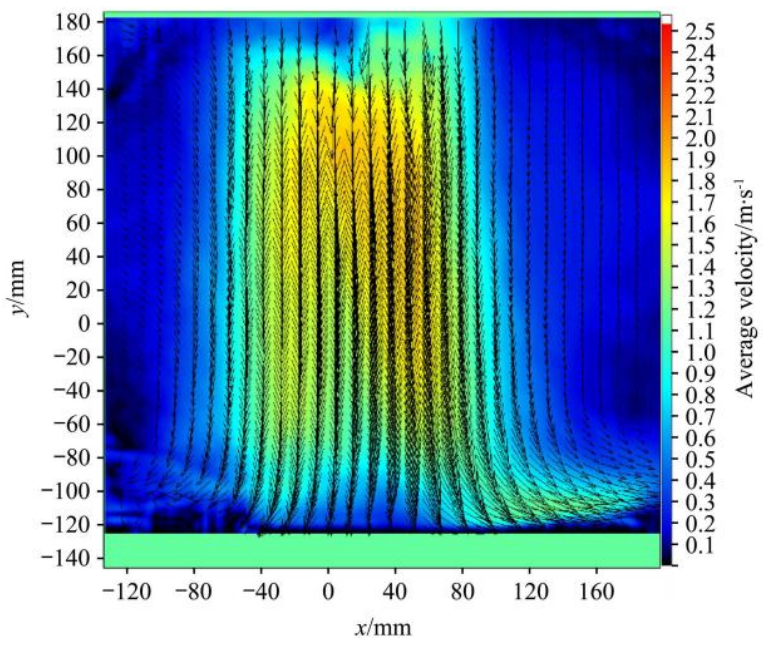

c. Central part of the $500 \mathrm{~mm}$ tests of the scaled-down drone 

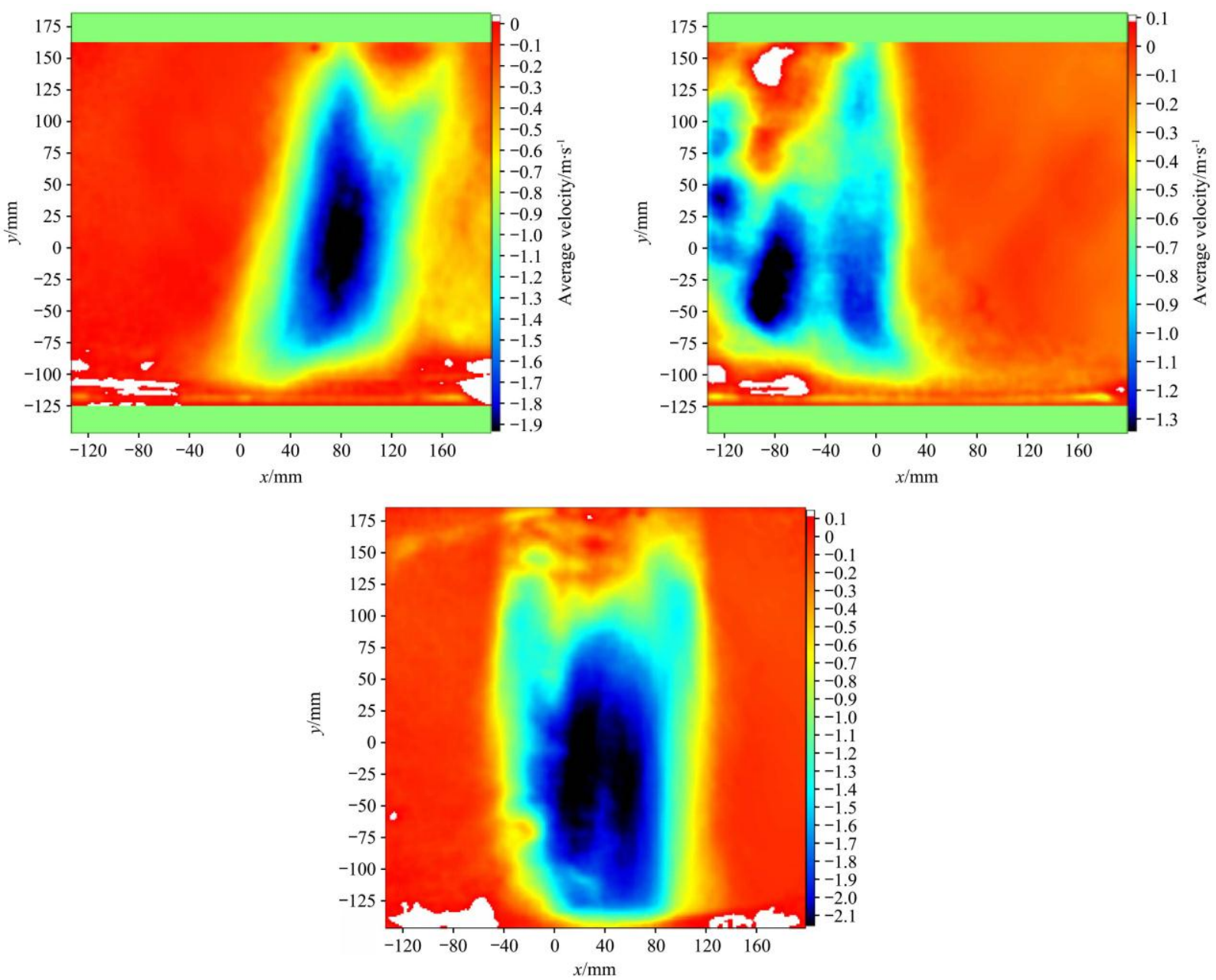

d. Average velocity of $V_{x}$ in the $500 \mathrm{~mm}$ tests of the scaled-down drone

Figure 12 Results of the $500 \mathrm{~mm}$ PIV tests of the scaled-down drone

According to Figures 11 and 12, it is known that the airflow was downward flowing with the concave shape of the flowing boundary. The velocity of the left and right parts was not symmetrically distributed. The maximum downwash airflow speed was about $2 \mathrm{~m} / \mathrm{s}$, which occurs in the surrounding area below the UAV. Instead, the central airflow speed of downwash was less than $1.5 \mathrm{~m} / \mathrm{s}$, and the transverse velocity profile below the UAV appears a double-peak scenario. Meanwhile, according to the average velocity of $V_{x}$, the downward motion of airflow was caused by the velocity in the negative $X$ direction, as the center of the downwash was less than zero; positive $V_{x}$ had contributions on the airflow periphery, which might result in the air diffusing outwards or turbulences.

Based on Figure 6, Figure 11 and Figure 12, Table 3 was summarized to show the velocity comparison by the CFD models of the scaled-down drone and the PIV tests. The results of the PIV tests were used as the standard to calculate the relative error of the maximum velocity.

Table 3 Velocity comparison by the PIV tests and the CFD models of the small UAV

\begin{tabular}{ccccc}
\hline $\begin{array}{c}\text { Height } \\
/ \mathrm{mm}\end{array}$ & $\begin{array}{c}\text { ANSYS } \\
/ \mathrm{m} \cdot \mathrm{s}^{-1}\end{array}$ & $\begin{array}{c}\text { PIV tests } \\
/ \mathrm{m} \cdot \mathrm{s}^{-1}\end{array}$ & $\begin{array}{c}\text { Relative error of } \\
\text { max. velocity/\% }\end{array}$ & Remarks \\
\hline 200 & $0-1.9$ & $0-1.7$ & 11.76 & PIV results as standard \\
500 & $0-1.8$ & $0-1.9$ & 5.26 & PIV results as standard \\
\hline
\end{tabular}

According to Table 3, the relative error of the CFD models of the scaled-down drone was less than $12 \%$, indicating that the CFD models had a high consistency with the real situations.
Furthermore, based on Figure 6 and Figure 12, Table 4 was summarized to show the velocity comparison between the CFD models of the real-used UAV and ten-time scaled results of the PIV tests. To do this size extension, UAV should be forced hovering and the rotor thrust coefficient $C_{T}$ is considered as a constant in both cases. The rotor trust is

$$
T=C_{T} \rho A \omega^{2} R^{2}
$$

where, $\rho$ is the air density, $\mathrm{kg} / \mathrm{m}^{3} ; A=\pi R^{2}$ is the rotor disc area, $\mathrm{m}^{2}$; $\omega$ is the rotating speed of the rotor, rpm; $R$ is the radius of the rotor, $\mathrm{m} ; C_{T}$ is a constant.

Thus, in terms of the same rotation speed $\omega$, the reference hover induced velocity, $v_{h}$, is proportional to the size of the UAV, $R$.

$$
v_{h}=\sqrt{\frac{T}{2 \rho A}}=\omega R \sqrt{\frac{C_{T}}{2}}
$$

According to Table 4, the relative error of the CFD models of JF01-10 was less than $34 \%$, identifying that the velocity prediction based on the Analogy of model migration should be further discussed.

Table 4 Velocity comparison by the PIV tests and the CFD models of JF01-10

\begin{tabular}{ccccc}
\hline $\begin{array}{c}\text { Height } \\
/ \mathrm{mm}\end{array}$ & $\begin{array}{c}\text { ANSYS } \\
/ \mathrm{m} \cdot \mathrm{s}^{-1}\end{array}$ & $\begin{array}{c}\text { Ten-time scaled } \\
\text { PIV tests } / \mathrm{m} \cdot \mathrm{s}^{-1}\end{array}$ & $\begin{array}{c}\text { Relative error of } \\
\text { Max. velocity } / \%\end{array}$ & Remarks \\
\hline 2000 & $0-12.43$ & $0-17$ & 26.88 & $\begin{array}{c}\text { PIV results as } \\
\text { standard } \\
\text { PIV results as } \\
\text { standard }\end{array}$ \\
\hline
\end{tabular}




\section{Discussion}

It is very difficult to directly verify the downwash CFD models of a real-used UAV, due to the large field size of downwash. Although wind-speed arrays can demonstrate downwash wind distribution, they cannot show flowing trajectories in detail. Thus, this paper proposed model migration to not only verify downwash CFD details but also avoid direct verification of real-used UAVs. According to the CFD models and the trials, the flowing trend of the CFD models could be verified by the PIV tests, which means that the CFD models are able to be used for the prediction of flowing and distribution of downwash. In addition, it could be illustrated that model migration is able to be applied for the demonstration of entire features and characteristics of downwash, including flow direction, distribution and airflow speed. Thus, model migration is more efficient and effective to obtain multi-factors at once than water-sensitive papers or wind-speed arrays (mentioned in Section 1).

Furthermore, a $20 \%$ even $25 \%$ error range was acceptable in several CFD validation studies ${ }^{[24,25]}$. Thus, compared with all the ANSYS CFD models and the PIV tests, it could be indicated that the relative error of the scaled-down drone was acceptable and CFD had a good ability of prediction. However, the relative error of the real-used UAV, JF01-10, exceeded the acceptance. Three factors might be considered. First, the body structure of the scaled-down drone is not completely the same as that of JF01-10, the real-used one. Second, the parameters of the propellers between the scaled-down drone and JF01-10 were a little bit different, although it was tried the best to keep the characteristics the same. For instance, the angle of attack may affect flight speed $^{[26]}$ and lift variation ${ }^{[27]}$, while these two types of UAVs are originally different on lift and load due to the distinct circumstance applied to. Third, CFD itself has a series of numerical errors from meshing methods to computation ${ }^{[28]}$. The method of CFD modelling is generally correct, but the meshes in complex areas may need to be further optimized Therefore, the error of velocities of the JF01-10 CFD models and the PIV tests might be significant.

According to the previous reference, the scaled-down UAV should be optimized by aerodynamic performance. Therefore, Equation (7) can be used for finding the relation among the angle of attack, heights and the aerodynamic parameter, and the linearity $R$ can be obtained. Due to the fixed flight height and angle of attack during tests, the real UAV can be used to obtain its aerodynamic parameter, so the calculated parameter can be substituted into Equation (7) to design the angle of attack of the scaled-down UAV.

$$
\left\{\begin{array}{l}
C_{n}=D C_{b}+d \\
R^{2}=1-\frac{\sum_{j=1}^{m}\left(\widehat{C}_{n j}-C_{n j}\right)^{2}}{\sum_{j=1}^{m}\left(\widehat{C}_{n j}-\bar{C}_{n j}\right)^{2}} \\
C_{n}=\frac{\xi}{\sqrt{R e}}+\zeta
\end{array}\right.
$$

where, $C_{n}$ is the aerodynamic parameter; $R$ is the linearity; $D$ and $d$ are constant; $\bar{C}_{n}$ is the means of the aerodynamic parameter; $\widehat{C}_{n}$ is the fitted aerodynamic parameter; $m$ is the number of fitted aerodynamic parameters; $R e$ is Reynolds and $\xi$ and $\zeta$ are constant.

Thus, for further study of CFD models and verifications of downwash based on model migration, three-dimensional (3D) scanning and 3D printing could be used to scale down JF01-10 and its propeller. However, because of the limit of both funds and available devices, they were not applied in this study. In addition, the mesh of complex positions of 3D real-used models should be carefully optimized to reduce numerical errors.

\section{Conclusions}

The method of model migration was proposed to develop CFD models and conduct verification trials. ANSYS was used to develop the CFD models of a real-used UAV, JF01-10, and a 1:10 scaled-down small drone. Particle image velocimetry (PIV) was the basic measurement device throughout the tests and the small drone was utilized to verify the method. Results showed that the relative error between the PIV tests and the CFD models of the small UAV was less than $12 \%$, while that between the tests and the CFD models of JF01-10 was less than $34 \%$, which means that model migration can be used to investigate multiple characteristics of downwash but needs to be further specifically optimized. This study is a preliminary but significant attempt to demonstrate CFD models and validation of agricultural UAVs, to be able to offer an inspiring of verification.

\section{Acknowledgements}

The authors acknowledge that this work was financially supported by the National Key Research and Development Program of China (2018YFD0700603 and 2016YFD0200702), National Natural Science Foundation of China (31701315), Independent Innovation Foundation for the Graduates of China Agricultural University (2020XYZC38A) and Chinese Universities Scientific Fund (2020TC116). The devices used in the paper were supported by National Research Center of Intelligent Equipment for Agriculture.

\section{[References]}

[1] Lan Y B, Chen S D, Fritz B K. Current status and future trends of precision agricultural aviation technologies. Int J Agric \& Biol Eng, 2017; 10(3): 1-17.

[2] Li J Y, Lan Y B, Shi Y Y. Research progress on airflow characteristics and field pesticide application system of rotary-wing UAV. Transactions of the CSAE, 2018; 34(12): 104-118. (in Chinese)

[3] Wang G B, Lan Y B, Qi H X, Chen P C, Hewitt A, Han Y X. Field evaluation of an unmanned aerial vehicle (UAV) sprayer: effect of spray volume on deposition and the control of pests and disease in wheat. Pest Management Science, 2019; 75(6): 1546-1555.

[4] Liao J, Zang Y, Luo X W, Zhou Z Y, Lan Y B, Zang Y, et al Optimization of variables for maximizing efficacy and efficiency in aerial spray application to cotton using unmanned aerial systems. Int J Agric \& Biol Eng, 2019; 12(2): 10-17.

[5] Wang J, Lan Y B, Zhang H H, Zhang Y L, Wen S, Yao W X, et al. Drift and deposition of pesticide applied by UAV on pineapple plants under different meteorological conditions. Int J Agric \& Biol Eng, 2018; 11(6): $5-12$.

[6] Meng Y H, Lan Y B, Mei G Y, Guo Y W, Song J L, Wang Z G. Effect of aerial spray adjuvant applying on the efficiency of small unmanned aerial vehicle on wheat aphids control. Int J Agric \& Biol Eng, 2018; 11(5): 46-53.

[7] Tang Y, Hou C J, Luo S M, Lin J T, Yang Z, Huang W F. Effects of operation height and tree shape on droplet deposition in citrus trees using an unmanned aerial vehicle. Computers and Electronics in Agriculture, 2018; 148: 1-7.

[8] Wang C L, He X K, Wang X N, Wang Z C, Wang S L, Li L L, et al. Testing method and distribution characteristics of spatial pesticide spraying deposition quality balance for unmanned aerial vehicle. Int J Agric \& Biol Eng, 2018; 11(2): 18-26.

[9] Zheng Y J, Yang S H, Zhao C J, Chen L P, Lan Y B, Tan Y. Modelling 
operation parameters of UAV on spray effects at different growth stages of corns. Int J Agric \& Biol Eng, 2017; 10(3): 57-66.

[10] Ay F, İnce G. Application of pesticide using unmanned aerial vehicle. In: 23nd Signal Processing and Communications Applications Conference (SIU), IEEE, 2015; pp.1268-1271.

[11] Salyani M, Fox R D. Performance of image analysis for assessment of simulated spray droplet distribution. Transactions of the ASAE, 1994; 37(4): 1083-1089.

[12] Zhang B, Tang Q, Chen L P, Xu M. Numerical simulation of wake vortices of crop spraying aircraft close to the ground. Biosystems Engineering, 2016; 145: 52-64.

[13] Yang F B, Xue X Y, Cai C, Sun Z, Zhou Q Q. Numerical Simulation and analysis on spray drift movement of multirotor plant protection unmanned aerial vehicle. Energies, 2018; 11(9): 1-20.

[14] Wang L, Chen D, Zhang M C, Wang Y, Yao Z, Wang S M. CFD Simulation of low-attitude droplets deposition characteristics for UAV based on multi-feature fusion. IFAC-PapersOnLine, 2018; 51(17): 648-653.

[15] Li J Y, Shi Y Y, Lan Y B, Guo S. Vertical distribution and vortex structure of rotor wind field under the influence of rice canopy. Computers and Electronics in Agriculture, 2019; 159: 140-146.

[16] Tan F, Lian Q, Liu C L, Jin B K. Measurement of downwash velocity generated by rotors of agriculture drones. Inmateh-Agricultural Engineering, 2018; 55(2): 141-150

[17] Zhang H, Qi L J, Wu Y L, Liu W W, Cheng Z Z, MUSIU E. Spatial-temporal distribution of down-wash airflow for multi-rotor plant protection UAV based on porous model. Transactions of the CSAE, 2018 50(2): 112-122. (in Chinese)

[18] Chen S D, Lan Y B, Brandley K F, Li J Y, Liu A M, Mao Y D. Effect of wind field below rotor on distribution of aerial spraying droplet deposition by using multi-rotor UAV. Transactions of the CSAM, 2017; 48(8): 105-113. (in Chinese)
[19] Tang Q, Zhang R R, Chen L P, Xu M, Yi T C, Zhang B. Droplets movement and deposition of an eight-rotor agricultural UAV in downwash flow field. Int J Agric \& Biol Eng, 2017; 10(3): 47-56.

[20] Wang H L, Li Q D, Ren Z, Zhao Q L, Dong X W, Liu F. Modeling of hypersonic vehicle via model migration method. Journal of Beijing University of Aeronautics and Astronautics, 2016; 42(12): 2640-2647. (in Chinese)

[21] Doty M J, Brooks T F, Burley C L, Bahr C J, Pope D. Jet noise shielding provided by a hybrid wing body aircraft. International Journal of Aeroacoustics, 2018; 17(1-2): 135-158.

[22] Raffel M C, Willert C E, Kompenhans J. Particle image velocimetry: A practical guide. 2nd Edition, Springer Berlin Heidelberg, 2007; 448p.

[23] Zheng Y J, Yang S H, Liu X X, Wang J, Norton T, Chen J, et al. The computational fluid dynamic modeling of downwash flow field for a six-rotor UAV. Frontiers of Agricultural Science and Engineering, 2018; 5(2): 159-167.

[24] Zhang Y N, Ke C F, Gao Y N, Liu S Y, Pan Y Y, Zhou N, et al. Syngas production from microwave-assisted air gasification of biomass: Part 2 model validation. Renewable Energy, 2019; 140: 625-632.

[25] Khalilzadeh A, Ge H, Ng H D. Effect of turbulence modeling schemes on wind-driven rain deposition on a mid-rise building: CFD modeling and validation. Journal of Wind Engineering and Industrial Aerodynamics, 2019; 184: 362-377.

[26] Thumthae C, Chitsomboon T. Optimal angle of attack for untwisted blade wind turbine. Renewable Energy, 2009; 34(5): 1279-1284.

[27] Cummings R M, Forsythe J R, Morton S A, Squires K D. Computational challenges in high angle of attack flow prediction. Progress in Aerospace Sciences, 2003; 39(5): 369-384

[28] Tyson W C, Roy C J. A higher-order error estimation framework for finite-volume CFD. Journal of Computational Physics, 2019; 394: 632-657. 